\section{Newborn and childhood screening programmes: criteria, evidence, and current policy}

\section{A C Elliman, C Dezateux, H E Bedford}

"The systematic application of a test or enquiry, to identify individuals at sufficient risk to benefit from further investigation or direct preventive action, amongst persons who have not sought medical attention on account of symptoms of that disorder" (Wald')

S creening is offered to apparently vell individuals to identify those at high risk of a specific condition, for whom early treatment, more effective treatment, or information may be offered in order to improve health outcome or to provide opportunities for informed decision making. By definition, screening tests are not diagnostic tests and therefore cannot separate reliably those with a specific condition from those without. Hence, those with a negative screening result will include some affected individuals, usually referred to as "false negatives", who may be falsely reassured by the screening result. Conversely, those with a positive screening result will include unaffected individuals, usually referred to as "false positives", who may be worried unnecessarily or be exposed to the risks of subsequent diagnostic tests. Therefore the threshold for a positive test will be determined by balancing the goals of screening and the perceived disbenefits of missing affected individuals or falsely labelling healthy individuals. $^{2}$

In the past screening tests have often been introduced as a result of the enthusiasm of one or more individuals without evidence of effectiveness or careful consideration of the wider implications of false reassurance or false labelling. In the $\mathrm{UK}$, this has led to the refinement of the historic "Wilson and Jungner" criteria," so that all proposed screening programmes are assessed against specified criteria. $^{4}$

\section{THE PRESENT FRAMEWORK}

To introduce a more systematic approach, the National Screening Committee (NSC) was established in 1996 with the remit that it should advise the Minister of State for Health on screening issues. Even though there are now separate health administrations, the NSC still has a role across the whole of the UK. One of the first tasks of the NSC was to define criteria for an acceptable screening programme. In recognition that the whole programme and not just the screening test has to be acceptable, there are now 19 criteria, although all may not have to be met for each programme. They cover the condition, the test, the treatment, and broader aspects of the programme. ${ }^{4}$ These criteria provide a framework for appraising evidence in relation to current or proposed screening programmes, which in turn form the basis of policy recommendations. For a current programme, these may include recommendations to continue screening, perhaps with modifications, or to discontinue the programme. For a proposed programme, these may include advice either to implement, or not to introduce. It is not within the remit of the committee to advise on issues to do with screening of particular high risk groups, for example, screening for hearing loss or hypothyroidism in children with Down's syndrome.

In 1998, Antenatal and Child Health Subgroups (ANSG and CHSG) were set up, in recognition of the large number of screening tests that are offered during pregnancy and early childhood. The CHSG advises the UK NSC on three main aspects of screening programmes: first, on the implementation, development, review, modification, and where necessary, cessation of UK childhood screening programmes; second, on the need for research in relation to screening, and for analytical work to help focus and make best use of research; and third, on quality management issues. In its first two years, the CHSG considered many conditions, often by holding workshops with relevant professionals and parent groups. Where relevant, these were held jointly with the ANSG to reflect the fact that screening strategies for some conditions could be offered before conception, during pregnancy, or after birth, albeit with often different goals. There is not room to describe all the recommendations in

\section{PHENYLKETONURIA,} CONGENITAL HYPOTHYROIDISM, AND MEDIUM CHAIN ACYL COA DEHYDROGENASE DEFICIENCY

In the UK, screening for phenylketonuria was introduced in 1969 and, for congenital hypothyroidism, in 1981. These programmes have been successful in identifying infants with phenylketonuria or congenital hypothyroidism before irreversible neurological damage has occurred, thereby preventing lifelong disability. ${ }^{56}$ The advent of tandem mass spectrometry (MS/MS) enables many compounds, including amino acids, to be assayed on the dried blood spots. In the $\mathrm{UK}, \mathrm{MS} / \mathrm{MS}$ is being increasingly used to screen for phenylketonuria, replacing older methods, and has the capacity to detect many other compounds. These include acyl carnitines which may be used to identify other inborn errors of metabolism, such as organic acidaemias and disorders of fatty acid oxidation, of which medium chain acyl CoA dehydrogenase deficiency (MCADD) is one of the most important. In appraising the criteria for MCADD screening, the CHSG has drawn on primary research carried out in the UK, ${ }^{7-9}$ systematic reviews commissioned by the UK NHS Research and Development programme, ${ }^{10} 11$ and reports from established or pilot programmes in North America, Australia, and Europe..$^{12-15}$ Despite international experience of screening well over a million newborn infants, important questions and uncertainties remain about performance and outcome of newborn screening for MCADD. In particular, there has been no report of a systematic follow up of longer term outcome in affected infants detected by screening. In view of this, the NSC recommended that primary research should be carried out within a UK setting to determine programme performance and early outcome.

\section{NEONATAL SCREENING FOR SICKLE CELL DISEASE}

Sickle cell disease predominantly, though not exclusively, affects particular ethnic minority populations in the UK. There is evidence from non-UK populations that prophylactic penicillin given following early diagnosis can reduce invasive infections with encapsulated organisms, in particular the pneumococcus, and reduce mortality. In the light of this evidence and the findings of two commissioned systematic reviews, ${ }^{16}{ }^{17}$ the NSC has recommended newborn screening for sickle cell disorders and antenatal screening for haemoglobinopathies, including thalassaemia. This 
was announced as a government priority in the NHS plan and details of the implementation are currently being considered by a working party.

\section{CYSTIC FIBROSIS}

A systematic review of screening for cystic fibrosis, commissioned by the NHS Research and Development programme, concluded that "the ability of [newborn] screening to alter prognosis has not been conclusively proven", that evidence available was "either predominantly short-term or subject to strong statistical bias", but that there was "some circumstantial evidence favouring a benefit". ${ }^{18}$ At the same time, the authors concluded that antenatal screening should be offered to pregnant women using a DNA based test with the aim of identifying couples with a 1 in 4 risk of an affected child, who could then be given information regarding their risk and reproductive choices. The NSC reviewed the evidence from this and other international reviews, from information published subsequently, and from workshops and meetings with relevant professional and parent groups. The NSC concluded that there was currently insufficient evidence of longer term benefit, specifically in relation to pulmonary function, to support implementation of newborn screening. Subsequently, a ministerial decision was made in July 2001 to formally introduce screening in England, followed shortly by a similar decision for Scotland. An implementation plan for England is being formulated. Currently antenatal screening is only carried out by a single centre in Scotland, but this policy is currently under active review.

\section{DUCHENNE MUSCULAR DYSTROPHY}

There is no treatment for Duchenne muscular dystrophy. The main value of screening is therefore to warn parents of the possibility that future boys in the family may be affected. ${ }^{19}$ This allows for the opportunity for prenatal counselling. Further evidence will become available from the experience of screening programmes in Wales, but, at present, there is not enough evidence to support a recommendation that screening should be universal.

\section{DEVELOPMENTAL DYSPLASIA AND CONGENITAL DISLOCATION OF THE HIP}

The term developmental dysplasia of the hip (DDH) refers to a spectrum of developmental hip disorders which include partial or complete displacement of the femoral head from the acetabulum, previously called congenital dislocation of the hip. This spectrum also includes acetabular dysplasia with or without displacement. Developmental displacement is associated with impaired growth and development of the hip and, usually, with subsequent abnormalities of gait and premature degenerative changes in the hip joint. A national screening programme based on clinical screening to identify neonatal hip instability was introduced in the UK in $1969 .^{20}$ There is however scientific uncertainty about the effectiveness of this programme, reflecting the fact that it has never been assessed in a randomised trial. Since the mid 1980s there has been increasing use of ultrasound to image newborn infants hips, although in the UK this has been principally offered to those with risk factors or clinically detected hip instability. While neither clinical nor ultrasound screening programmes have been satisfactorily evaluated in relation to longer term outcomes, evidence suggests that universal ultrasound programmes identify similar proportions of affected children compared with screening by expert clinical examiners. However, experience from other European countries with universal ultrasound screening programmes suggests that uncertainties in the ultrasound criteria for treatment result in very high treatment rates. This raises concerns about over treatment of infants with false positive screening results. Sources of information for the policy review included research carried out for the MRC Working Party on Congenital Dislocation of the Hip, ${ }^{21-23}$ and systematic reviews from the USA and Canada. ${ }^{24}{ }^{25}$ The NSC has recommended that clinical screening should continue, but with renewed emphasis on the standards and training for the clinical screening examination, with ultrasound used to image infants with clinical hip instability and recognised risk factors for DDH. Two national workshops have been held to develop an implementation plan. However, the NSC recognises that these policies are based on limited research evidence and plans to keep this policy under review.

\section{HEARING IMPAIRMENT}

The authors of a recent systematic review of neonatal hearing screening to identify children with permanent congenital hearing impairment concluded that the current infant distraction test (IDT) fails to fulfil the criteria for a good screening test. ${ }^{26}$ Even in ideal circumstances the IDT has poor sensitivity and specificity and cannot identify children before the age of 7-9 months. As early identification results in better outcome for children, and targeted neonatal screening only detects about $50 \%$ of cases, it was recommended that universal neonatal screening should be introduced. The CHSG held two workshops and established a universal neonatal hearing screening steering group to consider the evidence, consult with both health professionals and parents, and take forward an action plan. Twenty pilot sites have been set up around the country as the first step in implementation of universal neonatal hearing screening. Screening in most of the pilot sites will be hospital based, but the effectiveness of screening in other settings will also be addressed.

\section{VISION DEFECTS}

Currently babies are examined at birth and at 6-8 weeks for evidence of ocular abnormality, predominantly congenital cataract. At subsequent ages, an attempt is made to detect strabismus, reduced visual acuity, and abnormalities of colour vision. The evidence in favour of most of these activities is surprisingly sparse. ${ }^{27}$ The NSC has recommended that the programme of screening for retinopathy of prematurity should continue, as should newborn screening for media opacities and other eye anomalies, and a repeat examination between 4 and 6 weeks. However, training and supervision must be improved. ${ }^{28}$ The NSC also recommended that orthoptists should screen children between 4 and 5 years of age, with the aim of testing all children by the age of 5, but that all other routine vision testing in children less than 5 should cease, as should the test for 7 year olds. The major resource implication of these proposals is that orthoptists will be undertaking more tests. The feasibility of this needs to be examined in more detail.

It is tempting to recommend that all other tests of vision in school aged children should be abandoned because the evidence in their favour is poor and one might rely on self referral to the local optician. However, as there is some evidence that vision screening may reduce, if not eliminate, social gradients in age at presentation with asymptomatic amblyopia, ${ }^{29}$ it is possible that discontinuation of existing programmes may result in increased health inequalities. Therefore all other tests of vision in school aged children should continue unchanged-for further details see the Health For All Children website (http:// health-for-all-children.co.uk).

\section{GROWTH DISORDERS}

There is a lack of formal evidence as to the value of routine growth monitoring; its use in detecting abnormal growth velocity has low sensitivity and specificity. ${ }^{30}$ In 1998 a multiprofessional group met to develop a consensus on this issue. At this meeting it was agreed that the potential benefits of growth monitoring include identification of chronic disorders, giving reassurance to parents, monitoring the health of the nation's 
children, and supporting future research. Weight monitoring in infancy and among older children does not fulfil the criteria for screening. Single height measurements, with a cut off point at the 0.4 th centile, come closest to satisfying the criteria for screening. It has been suggested that it is good practice to weigh infants and young children at routine contacts, for example, at 6-8 weeks and when they attend for immunisations..$^{30}$ It is recommended that height and weight should be measured at school entry. At no other age does the measurement of height satisfy the criteria for a screening test and so cannot be recommended as a routine in well children. Recently, attention has been drawn to the increasing prevalence of obesity; however, there is insufficient evidence to support the introduction of routine screening for obesity at any age in childhood. ${ }^{31}$

\section{DISORDERS OF DEVELOPMENT AND BEHAVIOUR}

The terms "developmental screening" and "developmental assessment" are often incorrectly used interchangeably. As a result of this lack of clarity, tests which may be useful as assessment tools have often drifted into use as screening tests. Law et al concluded that there was insufficient evidence to introduce screening tests for speech and language delay. ${ }^{32}$ A trial of a screening instrument for autism in toddlers- the CHAT testhas recently been completed, ${ }^{33}$ which has reported a low sensitivity. This test was performed at 18 months (norms are not available for other ages). Of the 38 children who matched the criteria for high risk for autism and who were re-screened a month later, 12 continued to meet the criteria on re-testing. At 42 months, 10 of these 12 had diagnoses on the autistic spectrum, one had language delay, and one was normal. However, the sensitivity was only $18 \%$. A satisfactory screening test for autism is therefore currently unavailable. A systematic review of randomised controlled trials of occupational therapy for developmental coordination disorders failed to provide evidence that occupational therapy improved the child's ability to function in everyday life. ${ }^{34}$ There is no evidence to support population screening for developmental delay.

\section{ASSURING QUALITY IN SCREENING PROGRAMMES}

Newborn and childhood screening programmes depend on high quality services, including appropriate information for families who are offered testing, to ensure that informed choices are made, and that care is delivered in an effective and timely fashion. A national audit of newborn screening for phenylketonuria and congenital hypothyroidism, ${ }^{35}$ together with a commissioned review of quality management in screening,${ }^{36}$ have resulted in the establishment of a UKwide National Newborn Screening Programme Centre. This centre started work in April 2002 and is overseeing all screening programmes based on testing the heel prick sample. The centre has a remit to monitor, and facilitate improvement in, the quality of newborn screening processes and their outcomes for parents and their babies. A key feature will be the integration of this quality assurance programme for newborn screening into mainstream NHS quality management through a national framework for standard setting, training, and performance measurement.

\section{CONCLUSION}

Screening tests are only one component of a properly coordinated screening programme and should not be introduced until there is evidence that the potential benefits of the whole programme outweigh the harms. Once in place, it is important that there is continued monitoring of the programme to ensure the maintenance of standards and that unanticipated problems are not arising.

Unless there are good reasons to the contrary, no new screening programmes should be introduced on a local rather than national basis, except as a time limited research project. The menu of potential screening programmes is constantly changing and new topics will be considered by the NSC in the future. It is only through this process of reviewing evidence against explicit criteria that we can ensure that the benefits of new programmes outweigh the harms.

Arch Dis Child 2002;87:6-9

\section{Authors' affiliations}

D A C Elliman, Department of Child Health, St George's Hospital, Tooting, London SW 17 OQT, UK

C Dezateux, H E Bedford, Centre for Paediatric Epidemiology and Biostatistics, Institute of Child Health, 30 Guilford Street, London WCl 1EH, UK

David Elliman chairs the Child Health SubGroup of the National Screening Committee; Helen Bedford and Carol Dezateux are members of this subgroup

Correspondence to: $\operatorname{Dr}$ D A C Elliman, Department of Child Health, St George's Hospital, Tooting, London SW17 OQT, UK; DavidElliman@compuserve.com

Accepted 5 April 2002

\section{REFERENCES}

Wald NJ. Guidance on terminology. J Med Screen 1994:1:76.

2 Ades AE. Evaluating screening tests and screening programmes. Arch Dis Child 1990;65:792-5.

3 Wilson JMG, Jungner G. Principles and practice of screening for disease. Geneva: World Health Organisation, 1968.
4 National Screening Committee. First report of the National Screening Committee. Health Departments of the United Kingdom, 1998.

5 Simons WF, Fuggle PW, Grant DB, et al. Educational progress, behaviour, and motor skills at 10 years in early treated congenital hypothyroidism. Arch Dis Child 1997;77:219-22.

6 Beasley MG, Costello PM, Smith I. Outcome of treatment in young adults with phenylketonuria detected by routine neonatal screening between 1964 and 1971. Q J Med 1994:87: 155-60.

7 Pollitt RJ, Leonard JV. Prospective surveillance study of medium chain acyl-CoA dehydrogenase deficiency in the UK. Arch Dis Child 1998;79:116-19.

8 Wilson CJ, Champion MP, Collins JE, et al. Outcome of medium chain acyl-CoA dehydrogenase deficiency after diagnosis. Arch Dis Child 1999:80:459-62.

9 Pourfarzam M, Morris A, Appleton M, et al. Neonatal screening for MCAD deficiency: support from a retrospective study. Lancet 2001;358: 1063-4.

10 Pollitt RJ, Green A, McCabe ERB, et al. Neonatal screening for inborn errors of metabolism: cost, yield and outcome. Health Technol Assess 1997;1.

11 Seymour CA, Chalmers RA, Addison GM, et al. Neonatal screening for inborn errors of metabolism: a systematic review. Health Technol Assess 1997; 1.

12 Liebl B, Fingerhut R, Roschinger W, et al. Model project for updating neonatal screening in Bavaria: concept and initial results (in German). Gesundheitswesen 2000;62:189-95

13 Andresen BS, Dobrowolski SF, O'Reilly L, et al. Medium-chain acyl-CoA dehydrogenase (MCAD) mutations identified by MS/MS-based prospective screening of newborns differ from those observed in patients with clinical symptoms: identification and characterization of a new, prevalent mutation that results in mild MCAD deficiency. Am J Hum Genet 2001;68:1408-18.

14 Carpenter K, Wiley V, Sim KG, et al. Evaluation of newborn screening for medium chain acyl-CoA dehydrogenase deficiency in 275000 babies. Arch Dis Child Fetal Neonatal Ed 2001;85:F105-F109.

15 Zytkovicz TH, Fitzgerald EF, Marsden D, et al. Tandem mass spectrometric analysis for amino, organic, and fatty acid disorders in newborn dried blood spots: a two-year summary from the New England Newborn Screening Program. Clin Chem 2001;47:1945-55.

16 Davies SC, Cronin E, Gill M, et al. Screening for sickle cell disease and thalassaemia: a systematic review with supplementary research. Health Technol Assess 2000;4:i-99.

17 Zeuner D, Ades AE, Karnon J, et al. Antenatal and neonatal haemoglobinopathy screening in the UK: review and economic analysis. Health Technol Assess 1999;3:i-186.

18 Murray J, Cuckle H, Taylor G, et al. Screening for cystic fibrosis. Health Technol Assess 1999;3:i-104.

19 Jarvinen O, Lehesjoki $A E$, Lindlof $M$, et al. Carrier testing of children for two X-linked diseases: a retrospective study of comprehension of the test results and social and psychological significance of the testing. Pediatrics 2000;106:1460-5.

20 Standing Medical Advisory Committee. Screening for the detection of congenital dislocation of the hip in infants. London: Department of Health and Social Security, 1969

21 Godward S, Dezateux C. Surgery for congenital dislocation of the hip in the UK as a measure of outcome of screening. Lancet 1998:351:1149-52.

22 Dezateux C, Brown J, Parnaby A, et al. Cost-effectiveness of current and alternative screening strategies for congenital dislocation of the hip. Arch Dis Child 2000;82 (suppl 1):A2.

23 Dezateux C, Elbourne D, Arthur R, et al, on behalf of the MRC UK Collaborative Hip Trial 
Group. The MRC Hip Trial: a multicentre randomised trial of ultrasound imaging in infants with clinical hip instability detected by screening. Arch Dis Child 2001;84:A1

24 Lehmann HP, Hinton R, Morello P, et al. Developmental dysplasia of the hip practice guideline: technical report. Committee on Quality Improvement, and Subcommittee on Developmental Dysplasia of the Hip. Pediatrics 2000; 105:E57.

25 Patel H. Preventive health care, 2001 update: screening and management of developmenta dysplasia of the hip in newborns. Can Med Assoc J 2001;164:1669-77.

26 Davis A, Bamford J, Wilson I, et al. A critical review of the role of neonatal hearing screening in the detection of congenital hearing impairment. Health Technol Assess 1997; 1:i-176.

27 Snowdon SK, Stewart-Brown SL. Preschool vision screening. Health Technol Assess 1997; $1: i-83$
28 Rahi JS, Dezateux C. National cross sectional study of detection of congenital and infantile cataract in the United Kingdom: role of childhood screening and surveillance. The British Congenital Cataract Interest Group. BM 1999;318:362-5.

29 Smith LK, Thompson JR, Woodruff G. Children's vision screening: impact on inequalities in central England. J Epidemiol Community Health 1995;49:606-9.

30 Hall DM. Growth monitoring. Arch Dis Child 2000;82:10-15.

31 Hall DM. Child Growth Foundation on the epidemic of obesity. http:// www.health-for-all-children.co.uk/pdf/ epidemic_obesity/epidemic_of_obesity.pdf (last accessed 5 December 2001).

32 Law J, Boyle J, Harris F, et al. Screening for speech and language delay: a systematic review of the literature. Health Technol Assess 1998;2:1-184
33 Baron-Cohen S, Wheelwright S, Cox A, et al. Early identification of autism by the CHecklist for Autism in Toddlers (CHAT). J R Soc Med 2000;93:521-5.

34 Panagiotopulu K. Systematic review of randomised controlled trials of occupational therapy for developmental co-ordination disorder. MSc dissertation. University of London, 1997.

35 Streetly A, Corbett V. The national newborn screening programme: an audit of phenylketonuria and congenital hypothyroidism screening in England and Wales. London: Department of Public Health Medicine, UMDS Guy's and St Thomas's Medical Schools, 1998

36 Balmer S, Bowens A, Bruce E, et al. Quality managment for screening: report to the National Screening Committee. Leeds: Nuffield Institute for Health, 2000.

\section{EUROPE CALLING}

\section{Shortage of vaccines: USA, Europe, who next?}

W are all aware of the merits of preventing infectious diseases by vaccines and many of us have witnessed impressive examples of successful immunisation strategies: for example, the global eradication of smallpox, the elimination of poliomyelitis in most countries of the world, and the substantial decline of invasive Hib disease in the western world.

However, safety questions raised about certain vaccines-whether true (intussusception associated with rotavirus vaccine) or false (autism due to MMR) - have challenged paediatricians. Nowadays, it frequently requires long discussions, pronounced powers of persuasion and patience to convince parents about the benefits of vaccines. As if this were not enough, a further crisis has arisen: vaccine supplies are running short and/or the range of available vaccines is shrinking in the USA and several European countries. According to the Centers for Disease Control and Prevention, 8 of the 11 routine childhood vaccines are currently in short supply in the US, namely diphtheria, tetanus, and pertussis (DTPa); measles, mumps, rubella (MMR); varicella; and the pentavalent pneumococcal conjugate. The large demand for polysaccharides to be included in the latter may even negatively influence the availability of the familiar

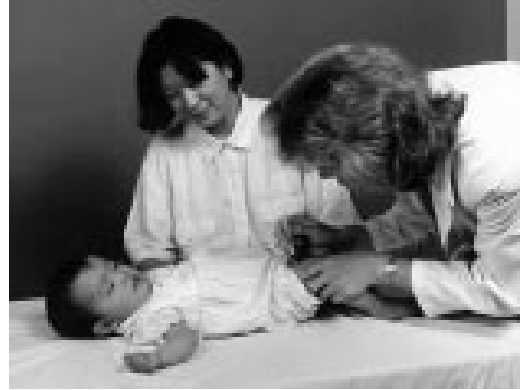

or subsidised vaccine manufacturers are increasingly being forced to show a profit. Unfortunately, inspections of some plants by regulatory authorities have revealed failures to maintain quality standards as set by GMP guidelines ("good manufacturing practice"). These have led to interruptions of production, thus intensifying the supply shortfall. Further, increasing numbers of different antigen components in modern vaccines make it more and more likely that a given production lot might not fulfil quality criteria for all ingredients.

What is the way out of this dilemma? Logically, the more manufacturers of standard vaccines there are, the less likely is significant shortage of supply. Furthermore, companies producing vaccines should be required to announce ahead of time any withdrawal of vaccines from the market thereby allowing other manufacturers to increase their output. States should consider long term contracts with vaccine manufacturers to assure sufficient supply for their population and, perhaps most importantly, local vaccine producers in less developed countries need to be strongly supported to maintain or increase their output with respect to quality and quantity. The children of the world (and their parents) deserve no less.

Ulrich Heininger European Editor 